\title{
Skin and mucous membranes' manifestations of dermatological diseases within the genital area in females
}

\author{
Katarzyna Plagens-Rotman'1, Renata Przybylska², Zygmunt Adamski ${ }^{3}$, Magdalena Czarnecka-Operacz ${ }^{3}$
}

'Department of Mother's and Child's Health, Poznan University of Medical Sciences, Poznan, Poland ${ }^{2}$ Department of Neurological Nursing, Poznan University of Medical Sciences, Poznan, Poland ${ }^{3}$ Department of Dermatology, Poznan University of Medical Sciences, Poznan, Poland

Adv Dermatol Allergol 2018; XXXV (2): 199-203

DOI: https://doi.org/10.5114/ada.2018.75243

\begin{abstract}
Introduction: Lichen sclerosus et atrophicus (LSA) and an inversed type of psoriasis belong to a group of benign dermatoses usually located within the region of female external genitalia. The most common subjective symptoms reported by patients are itching, pain and changes in the color and structure of the skin.

Aim: This paper presents 3 cases of patients suffering from selected dermatoses located within the external female genitalia treated at the Department of Dermatology, Poznan University of Medical Sciences.

Case reports: Case 1. A 78-year-old patient admitted to the Department of Dermatology diagnosed with lichen sclerosus and atrophic as well as scleroderma, which had already been confirmed by histopathological examination in 2014. Laboratory tests demonstrated an increased level of glycemia, elevated ESR and lymphopenia. In the treatment of TFX (Thymus factor $X$ ) therapy (immunomodulating treatment), vitamins A + E containing cream and Protopic $0.1 \%$ ointment twice daily were recommended. Case 2 . A patient aged 49 was admitted to the Department of Dermatology due to exacerbation of skin inflammation in the course of psoriasis. She presented with severe erythematous and papular lesions covered with silvery scales, with the highest intensity within the palmar surfaces of both hands, in the folds of under the breasts, groins, and therefore, the clinical picture was characteristic of inversed psoriasis (psoriasis inversa). Case 3. A 20-year-old patient admitted to the Department of Dermatology in order to proceed with the treatment of a diffuse type of scleroderma. Clinical diagnosis has been already confirmed by the skin biopsy (typical histological features of scleroderma), however exclusion of other dermatoses such as LSA was not possible.

Conclusions: While analyzing the available scientific reports, the physician in charge must adjust therapeutic options individually, taking into account the clinical condition of the patient in case of dermatological diseases within the female genital region.
\end{abstract}

Key words: lichen sclerosus and atrophic, psorasis inversa, treatment.

\section{Introduction}

Morphea, lichen sclerosus et atrophicus and inversed psoriasis (psoriasis inversa) are benign dermatoses located usually within the female external genitalia. The most frequent symptoms are itching, pain and discoloration as well as variations of the skin texture. Commonly used cosmetic substances as ingredients of various pharmaceuticals (e.g. fragrances) are considered to be important and potent predisposing factors for the onset of the contact skin reactions. Also dyes present in various fabrics and in fact all possibly irritant components present in common everyday use products should be considered in the same way. It is particularly crucial to emphasize that hypoestrogenism of the postmenopausal period and atrophy in the genital and lower urinary tract may also predispose to the whole variety of pathological changes within the vulvar area.

In case of patients with estrogen deficiency, epithelium lining of the vagina becomes thinner and the organ itself becomes less flexible. Moreover, there are atrophic changes in the form of flattened pubis and the reduction in body fat within the major labia as well as thinning of pubic hair is observed.

Address for correspondence: Katarzyna Plagens-Rotman PhD, Department of Mother's and Child's Health, Poznan University of Medical Sciences, 41 Jackowskiego St, 60-512 Poland, phone: +48 607165 279, e-mail: plagens.rotman@gmail.com Received: 18.02.2017, accepted: 20.06.2017. 


\section{Lichen sclerosus et atrophicus}

Lichen sclerosus et atrophicus (LSA) is one of the chronic inflammatory diseases usually diagnosed within the external genitalia. This kind of dermatosis can occur in virtually all age groups, but most cases concern the perimenopausal period $[1,2]$. Prevalence rate of LSA in the general population is estimated at $1: 300-1: 1000$; $1: 900$ for girls and $1: 30-40$ for women in the menopausal period. For comparison, in case of boys, the prevalence rate of LSA is estimated at $1: 160-220$, while for the adult male population, this number has not been evaluated [2].

Etiopathogenesis of LSA is not fully clarified, but the mechanisms of genetic autoimmune, infectious and hormonal background are highly possible [3-6]. Furthermore, factors affecting progression of the process are still unclear.

Thinning of the epidermis and hyperkeratosis of the skin of vulva is observed during the physical examination, and the results of histological examination reveal typical hyperplasia of the spinous layer and lymphocytic infiltration (lymphocyte CD4+ and CD8+), located initially in the upper layer of dermis and subsequently also in the deeper layers $[1,7]$.

Treatment of LSA is aimed at alleviation of subjective symptoms, reduction of inflammation and prevention of possible development of severe lesions of already malignant nature.

Therapeutic options include mainly topical preparations of corticosteroids (tGCS).

Clobetasol propionate in the form of $0.05 \%$ ointment is the first-line corticosteroid used in the treatment of LSA. Remission of symptoms and significant improvement in quality of life of patients is observed in 95\% of women whilst using the drug on a daily basis within 3 months $[8,9]$.

The preparations containing steroids in the composition, antifungal pharmaceuticals acting fungicidally - nystatin - and antibiotics - 3\% oxytetracycline - can be a valuable pharmacological medication to reduce the risk of progression of secondary bacterial infections and fungal infections.

However, any potential side effects of corticosteroids should be considered and the "benefit and risk" ratio should be carefully calculated before the decision is taken.

Table 1. Topical treatment of psoriasis inversed [18]

\begin{tabular}{ll}
\hline Recommended treatment & \multicolumn{1}{c}{ Alternative therapy } \\
\hline - Topical corticosteroids of & - Derivatives of vitamin A \\
small and medium potency & $(0.05 \%$ tazarotene $)$ \\
(short-term treatment) & - Derivatives of vitamin $\mathrm{D}_{3}$ \\
- Calcineurin inhibitors & \\
(tacrolimus and & \\
pimecrolimus) & \\
\hline
\end{tabular}

Topical calcineurin inhibitors (tCls) i.e. tacrolimus and pimecrolimus belong to a specific group of immunomodulatory/immunosuppressive medications.

By combining with macrophilin 12 they block the release of proinflammatory cytokines such as IL-2, IL-3, IL4, IL-5, tumor necrosis factor $\alpha$ (TNF- $\alpha$ ) and interferon- $\gamma$ $($ IFN- $\gamma)[10,11]$. Moreover, they block the granulocyte-macrophage colony-stimulating factor (GM-CSF). However, in the treatment of LSA surgical procedures and $\mathrm{CO}_{2}$ laser vaporization are not recommended due to the possibility of potential post-operative complications. Diagnosing with erosions, fistulas and areas of thinning skin during the clinical examination always indicates the necessity of biopsy of the skin due to the possible presence of a malignant tumor [12].

\section{Psorasis inversa}

Psoriasis is a chronic inflammatory skin disease which occurs in about 1-3\% of the general population. The etiology of psoriasis is still not fully understood, although genetic background as well as immunological disorders and environmental factors, such as traumas, bacterial and viral infections, alcohol, nicotine or stress play an essential role $[13,14]$. There are many types of psoriasis, and each particular type of pathogenesis is naturally distinct. Based on the contemporary medical knowledge, about $5-30 \%$ of psoriasis patients are further on diagnosed with psoriatic arthritis $[15,16]$. Moreover, a higher risk of developing hypertension, hyperlipidemia, obesity, diabetes and coronary artery in the patients is noticeable [17].

In case of the inversed type of psoriasis (psoriasis inversa), lesions are commonly located in the area of flexures, armpits, groins, in the folds of under the breasts [18]. Clinically the skin is smooth, shiny and devoid of scales - typical of the disease entity such as psoriasis. Treatment of psoriasis includes the use tGCS and tCls (Table 1) [18-20]. It is not recommended to use anthralin containing formulations (dithranol) and wood tar in the area of skin folds and flexures due to a high risk of severe irritation [18].

\section{Scleroderma (morphea)}

Scleroderma (morphea) is a rare autoimmune disease of connective tissue, affecting approximately 0.4$2.7 / 10,000$ of the global population. It is mainly diagnosed at the age ranging from 30 to 40 years $[21,22]$. The etiology of scleroderma has not been fully explained, but the autoimmune background is strongly emphasized, as well as mechanical or psychological trauma seems to be one of the most important triggering factors. Skin lesions characteristic for scleroderma are described as well-demarcated plaques with, sclerotic central foci of porcelainwhite color. Loss of skin appendages is also considered to be typical of the disease. In case of an active process, in 
the classical clinical cases, a lividly purple rim surrounding the sclerodermic center may be present [21]. Treatment of scleroderma [23, 24]:

- topical - glucocorticosteroids, vitamin $\mathrm{D}_{3}$ preparations, plasters containing prostaglandin PGE1, emollients;

- classical systemic treatment - anti-oxidants (piascledine, vitamin E), antibiotics (procaine penicillin, cephalosporins, minocycline), cyclosporine, cyclophosphamide, methotrexate, interferon $\gamma$, hydroxychloroquine and sulfones;

- phototherapy - UVA, UVA1, photochemotherapy, photopheresis;

- rehabilitation.

\section{Case reports}

\section{Case 1}

A 78-year-old female patient was admitted to the Department of Dermatology diagnosed with lichen sclerosus and atrophic as scleroderma, which had already been confirmed by skin biopsy in 2014. Duration of the disease ranged between 15 and 20 years. During the previous hospitalization in April 2016 laboratory diagnostic tests have been performed in relation to the diagnosed disease: anti-nuclear antibodies (ANA) in titer of 1/5120, Ro- 52+, RNP(+), SS-A (+). The direct immunofluorescence (direct immunofluorescence - DIF) showed single lumpy deposits of IgA (+/-) and C3 (+/-) along basement membrane zone (BZM, the basal membrane) and single IgM deposits under the epidermis. The patient was previously treated with procaine penicillin i.m., TNX, PUVA therapy, UVB311 and UVA 1 with a relatively good clinical effect (further phototherapy was rejected due to the implanted pacemaker. Starting from November 2013 onwards, the patient has been taking Disulone $(25 \mathrm{mg}$ daily) and she is regularly checked for the levels of methemoglobin and complete blood count as well as liver function parameters. Additionally, during the anamnesis with the patient: type 2 diabetes, hypertension, urinary incontinence, depression and hypothyroidism were mentioned. Laboratory results during hospitalization: increased levels of blood glucose (119 $\mathrm{mg} / \mathrm{dl})$, elevated erythrocyte sedimentation rate (ESR) $(30 \mathrm{~mm} / \mathrm{h})$ and lymphopenia $\left(0.94 \times 10^{3} / \mathrm{ml}\right)$. The following treatment was recommended: TFX $-10 \mathrm{mg}$ daily intramuscularly for 30 days followed by $20 \mathrm{mg} \times 1$ time per week vitamins $A+E$ containing cream (twice daily) and Protopic 0.1\% ointment (twice daily). During the check-up visit, a good clinical outcome has been recorded.

\section{Case 2}

A female patient aged 49 was admitted to the Department of Dermatology for the treatment of exacerbation in the course of psoriasis, earlier diagnosed as pustular psoriasis, generalized as well as psoriasis vulgaris. Five years of medical records were available. Recently hospitalized in the regional hospital (dermatological ward) in April 2016 also due to the exacerbation of the disease. A wide variety of diagnostic tests have been performed, such as chest X-ray (no perceptible lesions), abdominal ultrasound (liver of discreetly increased echogenicity, not enlarged, gall bladder with echoes of 2 calculi - the diameter of $6 \mathrm{~mm}, 2$ cysts in the left kidney - diameter of $10 \mathrm{~mm}$, one of them calcified), laryngological consultation (rhinorrhea infection), the levels of P-bodies anti-HCV, HBs Ag, HIV indicated (laboratory findings unremarkable). Systemic treatment with doxycycline $200 \mathrm{mg}$ intravenously once daily was used as well as classic topical treatment, which resulted in an improvement of the clinical status of the patient. On admission, erythematous and papular plaques covered with silvery scales were recorded, especially severe within palmar surfaces of hands, inframammary areas, groins and posterior rugae area. Therefore, the clinical picture has been considered to be characteristic for inversed type of psoriasis (psoriasis inversa). Additionally, the patient has already been diagnosed with bipolar disorder and remains under constant psychiatric supervision. Familial history for psoriasis was negative.

Laboratory tests carried out during the present hospitalization: eosinocytes $\left(0.55 \times 10^{3} / \mathrm{ml}\right)$, lymphocytosis (44.3\%) and neutrophilia (41\%), elevated levels of serum iron $(159 \mathrm{mg} / \mathrm{dl})$ and ASO (297 IU/ml).

Laboratory tests have not found contraindications to introduced systemic treatment with methotrexate or cyclosporine A. As a result of the systemic (Doxycycline 200 mg/day i.v., Trilac Plus) and topical treatment (5\% and $10 \%$ salicylic ointment, Acidum salicylicum, Clioquinol, Betamethasone gel) the dermatological condition of the patient has improved.

Indication for further treatment: systemic treatment: doxycycline (100 mg twice daily orally) in capsules for 7 days and Multilac (one capsule daily) for 7 days and the topical treatment of skin lesions in the areas under the breasts, groin and in the posterior rugae area: Protopic $0.1 \%$ ointment $2 \times$ daily for 4 weeks, subsequently $1 \times$ daily in the evening, then modification depending on the clinical condition; hands and feet: Advantan emulsion once daily, in the morning, 10\% urea ointment once daily in the evening and intensive skin moisturization (emollients e.g. Mediderma or Cerkobaza at least twice daily). In case of an unsatisfactory treatment effect, there are no contraindications for intensification of therapy (immunosuppression: cyclosporine A or methotrexate), as mentioned before.

\section{Case 3}

A 20-year old female patient was admitted to the Department of Dermatology for further diagnostic procedures in terms of multiple autoimmune connective tissue diseases as well as introduction of treatment of 
diffuse scleroderma, which has been already diagnosed and confirmed histologically. Medical records since 2008 were available. Skin lesions such as sclerosis and discoloration appeared in 2008, in January 2009 skin biopsy was performed revealing typical features for the diagnosis of morphea. Four courses of procaine penicillin treatment were applied with a very good clinical effect (nearly complete remission). Unfortunately in September 2015, sclerotic skin lesions within the area of calves, forearm, neck and chin have developed. Moreover, the patient reported periodic sensation of pressure/breathlessness in the area of neck as well as general weakness of the muscles mainly in the area of shoulder girdle.

Laboratory tests carried out during the present hospitalization, have not demonstrated any significant deviations from the normal ranges.

Capillaroscopy has been carried out - no pathological signs described (in terms of nail shafts of fingers II-V of hands) mainly vessels in the type of hairpins were visible. Tree-like vessels, enlarged, megakapilar, hemorrhages were not observed. MVD correct. Subpapillary plexus invisible. Pink background. Thickened stratum corneum).

Phototesting - lack of sensitivity to UVB broadband.

Abdominal ultrasound was performed (liver of regular echogenicity, not enlarged, without perceptible lesions, bile ducts not enlarged, gallbladder thin-walled, without echoes of calculi, pancreas not enlarged with normal echogenicity of the parenchyma, pancreatic duct not dilated. The structure and shape of the kidney regular, without stagnation and echoes of calculi. Spleen homogeneous, not enlarged. Retroperitoneal space and the large abdominal vessels sonographically correct, lymph glands not enlarged, bladder filled, thin-walled).

Chest X-ray - correct image. Contrast esophagus X-ray - without any pathological deviations.

Otolaryngological consultation (for ENT) (chronic tonsillitis) and dental consultation were also carried out potential foci of the disease in the oral cavity were not found.

Treatment with procaine penicillin - 2.4 million units for 10 days as well as the topical treatment - cream with vitamin $\mathrm{A}$ and $\mathrm{E}$.

\section{Conclusions}

At the beginning of the XXI century, diseases of vulva are still a significant challenge for dermatologists and gynecologists [25]. Non-specific manifestations of the diseases of vulva include such symptoms as itching, discoloration, and changes in the structure of vulva as well as pain. Positive allergic reactions are diagnosed in $26 \%$ to $80 \%$ of cases, usually they are reactions to cosmetics, substances included in medicines and preservatives. Among other allergens such irritants as fragrances, rubber and articles for daily use should be mentioned. More- over, an increased risk of irritation, mechanical damage and infection in postmenopausal women is observed.

Glucocorticoids used in the treatment of skin diseases are the first-line medications. The total or partial regression of symptoms was observed in 54-96\% of women diagnosed with LSA. However, a schedule of treatment clearly presented to the patient, which includes the amount of the drug used and the frequency of its use can affect the remission of the disease.

Tacrolimus and pimecrolimus belong to a group of immunosuppressants which are primarily anti-inflammatory. Moreover, inhibitors of calcineurin allow to avoid adverse effects (side effects) of glucocorticoids.

Finally, it is worth reminding generally applicable vulvar skin care principles.

Vulvar Skin Care Principles [1]:

- It is recommended that soap substitutes should be used to wash the area of vulva.

- The skin around the pubic area should be washed with water and a small amount of cream or ointment. Soap substitutes are safe even when used frequently;

- Showering is recommended instead of bath taking. It is absolutely recommended to add emollients to water while taking a bath.

- Washcloths and sponges are inadvisable in vulva washing.

- Gentle massaging of the vulva is desirable.

- Use a soft towel to pat dry the vulva.

- Close-fitting cotton or silky underwear is recommended.

- Sleeping without underwear is advisable.

- Do not use liquid fabric softener and enzyme washing powders.

- Using soap, shower gel, body scrubs, bubble bath, deodorant and wet wipes are inadvisable.

- Avoid frequent use of underwear inserts and sanitary napkins.

- Eliminate antiseptic (applied to the vulvar area as a cream or as a bath additive).

- Wearing white lingerie or underwear in bright colors is advisable. Dark fabric dyes (black, navy) can cause allergies.

- Avoid the use of colored toilet paper.

- In case of the tendency to scratching, nail painting is not recommended. Emollients:

- Using of emollients as moisturizers during the day is advisable.

- Daily application of moisturizers to relieve the discomfort.

- Finding a moisturizing substance which suits best.

- If the skin is irritated, cream for the care should be stored in the refrigerator and applied it chilled in order to soothe the skin as often as needed. 


\section{Conflict of interest}

The authors declare no conflict of interest.

\section{References}

1. Olek-Hrab K, Jenerowicz D, Osmola-Mańkowska A, et al. Selected vulvar dermatoses. Ginekol Pol 2013; 84: 959-65.

2. Cegielska A, Imko-Walczuk B, Jaśkiewicz J, et al. The role of genetic, autoimmune, infective and hormonal factors in pathogenesis of lichen sclerosus - review of the literature. Przegl Dermatol 2011; 98: 355-61.

3. Eisendle K, Grabner T, Kutzner H, et al. Possible role of Borrelia burgdorferi sensu lato infection in lichen sclerosus. Arch Dermatol 2008; 144: 591-8.

4. Zollinger T, Merts KD, Schmid M, et al. Borrelia in granuloma annulare, morphea and lichen sclerosus: a PCR-based study and review of the literature. J Cutan Pathol 2010; 37: 571-7.

5. Cooper S, Ali I, Baldo M, et al. The association of lichen sclerosus and erosive lichen planus of the vulva and autoimmune disease. Arch Dermatol 2008; 144: 1432-5.

6. Sherman V, McPherson T, Baldo M, et al. The high rate of familial lichen sclerosus suggests a genetic contribution: an observational cohort study. J Eur Acad Dermatol Venereol 2010; 29: 1031-4.

7. Carli P, Cattaneo A, Pimpinelli N, et al. Immunohistochemical evidence of skin immune system involvement in vulvar lichen sclerosus et atrophicus. Dermatologica 1991; 182: 18-22.

8. Cooper SM, Gao XH, Powell JJ, et al. Does treatment of vulvar lichen slerosus influence its prognosis? Arch Dermatol 2004; 140: 702-6.

9. Smoth YR, Quint EH. Clobetasol propionate in the treatment of premenarchal vulvar lichen sclerosus. Obstet Gynecol 2001; 98: 588-9.

10. Plagens-Rotman K, Przybylska R, Jacenko A, Czarnecka-Operacz $\mathrm{M}$. Therapeutic and nursing aspects of atopic dermatitis. In: Public health - the biggest topics. Adamek R, Maksymiuk T (eds). Wydaw. Nauk. Uniw. Med. im. K. Marcinkowskiego w Poznaniu, 2016; 163-76.

11. Sokołowska-Wojdyło M, Góralczyk A, Barańska-Rybak W, Szczerkowska-Dobosz A. Cancerogenic effect of topical calcineurin inhibitors - a real threat or invalid concerns? Przegl Dermatol 2012; 99: 625-31.

12. Bowen AR, Vester A, Marsden L, et al. The role of vulvar skin biopsy in the evaluation of chronic vulvar pain. Am J Obstet Gynaecol 2008; 199: 467-9.

13. Reich A, Wojcik-Maciejewicz A, Stominski AT. Stress and the skin. G Ital Dermatol Venereol 2010; 145: 213-9.

14. Mikiel D, Kapała W, Neneman A. Psoriasis as a problem in surgical practice. Pieleg Chirur Angiol 2016; 2: 64-9.

15. Eder L, Chandran V, Shen H, et al. Incidence of arthritis in a prospective cohort of psoriasis patients. Arthritis Care Res 2011; 63: 619-22.

16. Naredo E, Moller I, de Miguel E, et al. High prevalence of ultrasonographic synovitis and enthesopathy in patients with psoriasis without psoriatic arthritis: a prospective casecontrol study. Rheumatology (Oxford) 2011; 50: 1838-48.

17. Naldi L, Mercuri SR. Epidemiology of comorbidities in psoriasis. Dermatol Ther 2010; 23: 114-8.

18. Szepietowski J, Adamski Z, Chodorowska G. Diagnostics and treatment of psoriasis vulgaris: guidelines of the Polish Dermatological Society. Part I: mild psoriasis, psoriasis in children. Przegl Dermatol 2012; 99: 83-96.
19. Lebwohl M, Freeman A, Chapman MS, et al. Proven efficacy of tacrolimus for facial and intertriginous psoriasis. Arch Dermatol 2005; 141: 1154.

20. Brune A, Miller DW, Lin P, et al. Tacrolimus ointment is effective for psoriasis on the face and intertriginous areas in pediatric patients. Pediatr Dermatol 2007; 24: 76-80.

21. Bergler-Czop B, Brzezińska-Wcisło L, Meszyńska E. Bullous morphea - a case report. Przegl Dermatol 2010; 97: 390-4.

22. Baran A, Flisiak I, Chodynicka B. Rare coexistence of psoriasis, lichen sclerosus and morphea - case report and literature data. Przegl Dermatol 2012; 99: 39-44.

23. Schlaak M, Friedlein H, Kauer F, et al. Successful therapy of a patient with recalcitrant generalized bullous scleroderma by extracorporeal photophoresis and mycophenolate mofetile. J Eur Acad Derm Venereol 2008; 22: 631-3.

24. Appelhans C, Breuckmann F, Gambichler T, et al. Unilateral generalized morphea is a rare variant of localized scleroderma. Eur J Med Res 2006; 28: 152-6.

25. Białynicki-Birula R, Reszke R, Szepietowski JC. High-frequency ultrasonography (HFUS) as a useful tool in differentiating between plaque morphea and extragenital lichen sclerosus lesions. Adv Dermatol Allergol 2017; 34: 485-9. 